\title{
Neu aufgetretenes bullöses Pemphigoid bei einer Patientin mit COVID-19
}

\author{
Natalie Olson ${ }^{\mathrm{a}}$ David Eckhardt ${ }^{\mathrm{a}}$ Angela Delano $^{\mathrm{b}}$ \\ a Rocky Vista University, Parker, CO, USA; \\ bSt. Anthony North Family Medicine Residency Program, Denver, CO, USA
}

\section{Zusammenfassung}

Der vorliegende Artikel berichtet erstmals über ein bullöses pemphigoidartiges Exanthem in Verbindung mit COVID-19. In dieser Arbeit soll ein ungewöhnlicher dermatologischer Fall im Zusammenhang mit einer COVID-19-positiven Infektion vorgestellt werden, um die Virussymptomatik besser zu verstehen. Eine 37-jährige Frau mit Adipositas Grad 3, Diabetes mellitus Typ II und Hypertonie in der Vorgeschichte stellte sich im September 2020 in der Notaufnahme vor und wurde bis November 2020 ambulant und stationär behandelt. Nach Angaben der Patientin lagen bei ihr selbst oder in der Familie keine Hauterkrankungen vor. Die Patientin war vor der stationären Aufnahme positiv auf COVID-19 getestet worden und kam mit einem schweren persistierenden juckenden Exanthem, das die dermatopathologischen, serologischen und klinischen Kriterien für die Diagnose eines bullösen Pemphigoids erfüllte, ins Krankenhaus. Die histopathologische Untersuchung ei- ner HE-gefärbten Stanzbiopsie aus der Beugeseite des linken Handgelenks ergab eine epidermale Keratinozytennekrose, subepidermale Bläschenbildung mit Eosinophilen, zarte Stränge in der papillären Dermis sowie ein subepidermales Ödem. In der direkten Imunfluoreszenz-Stanzbiopsie aus der Beugeseite des linken Handgelenks zeigte sich eine starke lineare lgG-Färbung in der dermoepidermalen Junktionszone mit einer etwas schwächeren fokalen linearen C3-Färbung. Die antigenspezifische Serologie war mit einem bullösen Pemphigoid vereinbar. Bislang liegen keine Berichte über einen Zusammenhang zwischen einer COVID-19-Infektion und einem bullösen Pemphigoid an der Haut vor; daher stellt dieser Fall eine wichtige Ergänzung der Evidenzlage dar und trägt dazu bei, das bullöse Pemphigoid im Zusammenhang mit einer Virusinfektion zu identifizieren.

(c) 2021 Natalie Olson et al.

\section{Einleitung}

Im Jahr 2019 erschien in Wuhan, China, ein (neuartiges) Coronavirus auf der Bildfläche. Das Virus, das nachgewiesenermaßen schwere Pneumonien verursacht, hat seit seinem Auftreten 35 Millionen Menschen befallen und weltweit zu 1,5 Millionen Todesfällen geführt. Es handelt sich bei SARS-CoV-2 (severe acute respiratory syndrome coronavirus 2) um ein Einzelstrang-RNAVirus, das sich über Aerosoltröpfchen verbreitet und über den Angiotensin-Converting Enzyme 2 (ACE2)-Rezeptor in die Wirtszellen eindringt [1]. Nach allgemein akzeptiertem Verständnis weisen Typ-II-Alveolarzellen und sekretorische Zellen der Lunge eine hohe ACE2-Expression auf, doch wird vermutet, dass ACE2-Rezeptoren auch auf dem Dünndarmepithel, oralen
Epithelzellen, Endothelzellen und auf den Keratinozyten in der Haut zu finden sind [1]. Zwar zeigen die meisten symptomatischen Patienten, die mit dem Virus infiziert sind, grippeähnliche Symptome wie Fieber, Husten, Erschöpfung und Krankheitsgefühl, doch deuten aktuelle Studienergebnisse darauf hin, dass es bei einigen Patienten auch zu kutanen Manifestationen der Krankheit kommt. Es ist wichtig, auch an die dermatologischen Manifestationen von SARS-CoV-2 zu denken, da sie dem Arzt als Orientierungshilfe dienen können, um bei der Diagnosestellung COVID-19 von anderen Atemwegsviren abzugrenzen. In der vorliegenden Arbeit berichten wir über eine ungewöhnliche dermatologische Manifestation von bullösem Pemphigoid (BP) bei einer Patientin mit nachgewiesener COVID-19-Infektion. 

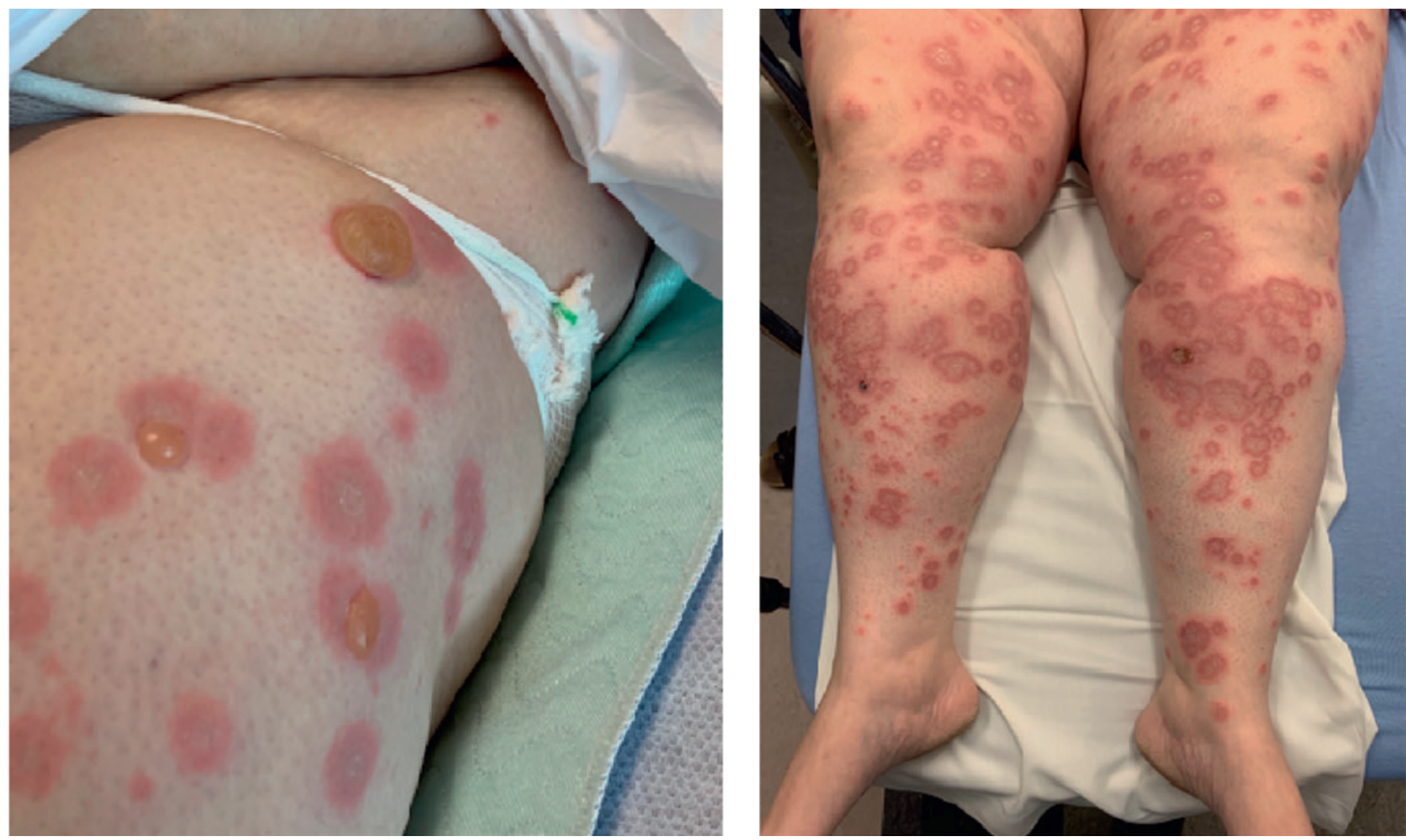

Abb. 1. (a) Linke Hüfte mit Blanching-Effekt und prall gespannten, berührungsempfindlichen Blasen. (b) Beidseitige untere Extremitäten mit juckenden erythematösen, schuppenden, anulären Läsionen in unterschiedlichen Krankheitsstadien.

\section{Fallbericht}

Bei unserer Patientin handelte es sich um eine 37-jährige Frau mit Diabetes mellitus Typ 2, Hypertonie und Adipositas Grad 3 in der Vorgeschichte, die sich im September 2020 mit einem Exanthem, das sich zunehmend verschlimmert hatte, in der Notaufnahme vorstellte. Am Tag der Aufnahme gab sie an, im Verlauf der vorangegangenen Woche die Entwicklung roter, erhabener Läsionen an ihren Armen bemerkt zu haben. Die Läsionen juckten leicht und waren nicht berührungsempfindlich. Am folgenden Tag traten die typischen COVID-19-Symptome (Fieber, Kurzatmigkeit und Muskelschmerzen) auf, und an Tag 3 nach Symptombeginn wurde sie positiv auf COVID-19 getestet. Bei der Patientin selbst oder in der Familie lagen keine Hautkrankheiten oder Autoimmunerkrankungen in der Vorgeschichte vor. Nach Angaben der Patientin hatte es in letzter Zeit keine Veränderungen ihrer Umgebung, Reinigungsmittel, Seifen oder Lotionen gegeben und es waren unlängst keine Impfungen oder Änderungen ihrer Medikamente erfolgt. Die Medikation bestand in Lisinopril $20 \mathrm{mg}$ einmal täglich seit Februar 2020 gegen die Hypertonie und Metformin 500 mg zweimal täglich seit Februar 2020 zur Behandlung des Typ-2-Diabetes.

In den zehn Tagen bevor sich die Patientin in der Notaufnahme vorstellte, hatte sich das Exanthem auf die unteren Extremitäten, den Rumpf und schließlich auf Hals und Kinn ausgebreitet. Die Läsionen wurden zunehmend größer und einige wurden zu Blasen. Die Schwere des Juckreizes und ihre Beschwerden waren der
Grund, dass sie die Notaufnahme aufsuchte. Sie war tachypnoeisch, jedoch unter Raumluft ausreichend oxygeniert. Zur Linderung der Tachypnoe erhielt sie zusätzlich 1-2 1 Sauerstoff über eine Nasenkanüle, wobei während des stationären Aufenthalts keine Hypoxie festgestellt wurde.

Auf Befragen berichtete die Patientin über ein negatives NikolskyZeichen, Blanching-Effekt (Weißfärbung der Haut), prall gespannte und berührungsempfindliche Blasen sowie schuppende nicht berührungsempfindliche Läsionen. Bei der körperlichen Untersuchung fand sich keine Schleimhautbeteiligung (Abb. 1). Die hämatologischen Untersuchungen der Patientin fielen normal aus. Bei der Aufnahme fanden sich eine leichte Hypokaliämie (die sich während des stationären Aufenthalts normalisierte), erhöhte Blutzuckerwerte (zwischen $134 \mathrm{mg} / \mathrm{dl}$ und $146 \mathrm{mg} / \mathrm{dl}$ im Serum und HbAlC-Wert von 5,8 \%), eine Hypoalbuminämie sowie erhöhte Leberenzyme (ALT und AST). Die angeforderte Bestimmung der D-Dimere und des C-reaktiven Proteins ergab ebenfalls erhöhte Werte. In den Abbildungen 2 (a) bis 2 (e) sind die Laborergebnisse der Patientin zum Zeitpunkt der Aufnahme dargestellt; abnorme Werte sind gelb hervorgehoben.

Tabelle 1 zeigt die Ergebnisse der Hautbiopsie, der direkten Immunfluoreszenz und der antigenspezifischen serologischen Untersuchung, die bei der Patientin durchgeführt wurden. Bilder der pathologischen Untersuchungen waren nicht verfügbar.

Differentialdiagnostisch kamen Erythema multiforme, Verätzung oder Exposition gegenüber Chemikalien, leukozytoklasti- 


\begin{tabular}{|c|c|c|c|c|c|}
\hline Component & Latest Ref Rng \& Units & 9/19/2020 & $9 / 20 / 2020$ & $9 / 21 / 2020$ & $9 / 22 / 2020$ \\
\hline WBC White Blood Count & $3.7-11.110^{*} 3 / \mathrm{LL}$ & 8.6 & 6.7 & 6.4 & 8.1 \\
\hline RBC Red Blood Count & $4.11 \cdot 5.6310^{\circ} 6 / \mu L$ & 4.85 & 4.44 & 4.26 & 4.52 \\
\hline HGB Hemoglobin & $11.9 \cdot 16.3 \mathrm{~g} / \mathrm{dL}$ & 14.4 & 13.2 & 12.7 & 13.3 \\
\hline HCT Hematocrit & $37.0-47.7 \%$ & 44.4 & 40.5 & 39.1 & 41.4 \\
\hline MCV Mean Cell Volume & $81.97 \mathrm{fL}$ & 92 & 91 & 92 & 92 \\
\hline MCH Mean Coll Hemoglobin & $26.7-33.1 \mathrm{pg}$ & 29.7 & 29.7 & 29.8 & 29.4 \\
\hline MCHC Mean Cell Hgb Conc & $31.1 .35 .3 \mathrm{~g} / \mathrm{dL}$ & 32.4 & 32.6 & 32.5 & 32.1 \\
\hline RDW-CV & $12.3 \cdot 15.9 \%$ & 13.6 & 13.6 & 13.6 & 13.3 \\
\hline RDW-SD & $37.1 \cdot 49.8 \mathrm{fL}$ & 46.8 & 46.3 & 46.4 & 45.2 \\
\hline PLT Platelet Count & $150 \cdot 40010^{*} 3 / \mathrm{\mu L}$ & 239 & 225 & 229 & 272 \\
\hline MPV Mean Platelef Volume & fl. & 10.2 & 10.1 & 10.0 & 10.3 \\
\hline Neutrophils \% & $\%$ & 75 & 64 & 55 & 76 \\
\hline Immature Granulocytes \% (Auto) & $\%$ & 0.3 & 0.6 & 0.5 & 0.7 \\
\hline Lymphocytes \% & $\%$ & 16 & 23 & 30 & 18 \\
\hline Monocyes \% & $\%$ & 4 & 5 & 6 & 5 \\
\hline Eosinophils \% & $\%$ & 4 & 6 & 8 & 1 \\
\hline Basophils \% & $\%$ & 0 & 0 & 0 & 1 \\
\hline Neutrophils Absolute & $1.70 \cdot 7.1010^{\star 2} 3 / \mathrm{uL}_{\mathrm{L}}$ & 6.45 & 431 & 3.52 & 6.13 \\
\hline Immature Granulocytes Absolute & $<0.110^{*} 3 / \mu \mathrm{L}$ & 0.03 & 0.04 & 0.03 & 0.06 \\
\hline Lymphosytes Absolute & $1.1 \cdot 3.710^{*} 3 / \mathrm{uL}$ & 1.4 & 1.6 & 1.9 & 1.4 \\
\hline Monocytes Absolute & $0.3 \cdot 0.910^{*} 3 / \mathrm{uL}$ & 0.4 & 0.4 & 0.4 & 0.4 \\
\hline Eosinophils Absolute & $0.0 \cdot 0.510^{\circ} 3 / \mathrm{uL}$ & 0.4 & 0.4 & 0.5 & 0.1 \\
\hline Basophils Absolute & $0.0 \cdot 0.110^{*} 3 / \mathrm{uL}$ & 0.0 & 0.0 & 0.0 & 0.0 \\
\hline Nucleated RBC & $0 / 100$ WBCs & 0 & 0 & 0 & 0 \\
\hline
\end{tabular}

(a)

\begin{tabular}{|l|l|l|}
\hline Component & Latest Ref Rng \& Units & 9/19/2020 \\
\hline D-dimer & $\leq=0.49 \mathrm{mg} / \mathrm{L}$ FEU & 5.85 (H) \\
\hline
\end{tabular}

(c)

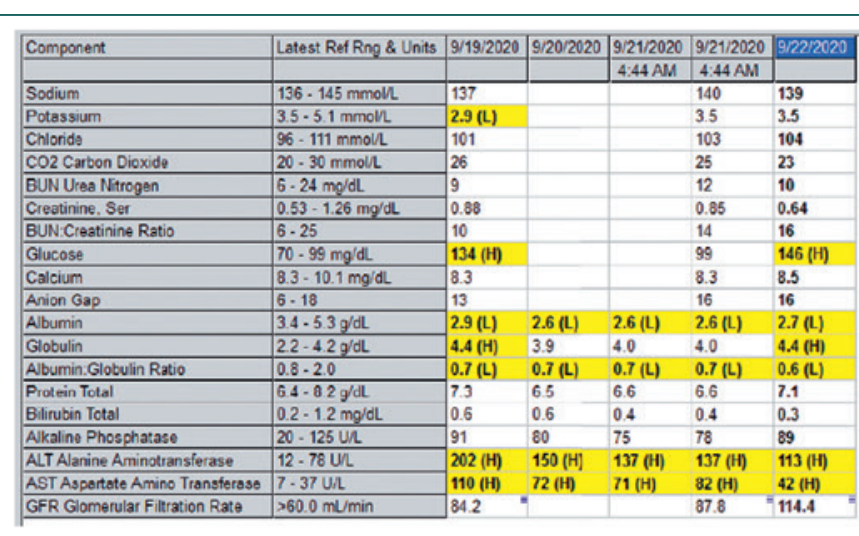

(b)

\begin{tabular}{|l|l|l|}
\hline Component & Latest Ref Rng \& Units & 9/19/2020 \\
\hline CRP C-Reactive Protein & $0.00-2.99 \mathrm{mg} / \mathrm{L}$ & $\mathbf{1 0 2 . 6 0}(\mathrm{H})$ \\
\hline
\end{tabular}

(d)

\begin{tabular}{|l|l|l|}
\hline Component & Latest Ref Rng \& Units & 9/21/2020 \\
\hline Hemoglobin A1C & $4.0-5.6 \%$ & $5.8(\mathrm{H})$ \\
\hline eAG Estimated Ave Glucose & $\mathrm{mg} / \mathrm{dL}$ & $\mathbf{1 2 0}$ \\
\hline
\end{tabular}

Abb. 2. Laborergebnisse. (a) Differentialblutbild. (b) Vollständiges Stoffwechselprofil. (c) D-Dimere. (d) C-reaktives Protein. (e) Hämoglobin $A_{1 c}$.

Tab. 1. Laborergebnisse der Patientin

\begin{tabular}{|c|c|c|c|}
\hline Diagnostik & Lokalisation/Typ & Ergebnis & Normalwert \\
\hline Biopsie & Beugeseite des linken Handgelenks & $\begin{array}{l}\text { Epidermale Keratinozytennekrose, subepidermale } \\
\text { Bläschenbildung mit Eosinophilen, zarte Stränge in } \\
\text { der papillären Dermis, subepidermales Ödem }\end{array}$ & $<20 \mathrm{RU} / \mathrm{mL}$ (negativ) \\
\hline $\begin{array}{l}\text { Direkte } \\
\text { Immunfluoreszenz }\end{array}$ & Beugeseite des linken Handgelenks & $\begin{array}{l}\text { Ausgeprägte lineare lgG-Färbung an der } \\
\text { dermoepidermalen Junktionszone, mit } \\
\text { schwächerer und fokaler linearer C3-Färbung }\end{array}$ & $\geq 20 \mathrm{RU} / \mathrm{mL}$ (positiv) \\
\hline $\begin{array}{l}\text { Antigen-spezifische } \\
\text { serologische Untersuchung }\end{array}$ & $\begin{array}{l}\text { Bullöses Pemphigoid } 180 \mathrm{lgG} \text { AK } \\
\text { Bullöses Pemphigoid } 230 \mathrm{lgG} \text { AK }\end{array}$ & $\begin{array}{l}211,4 \mathrm{RU} / \mathrm{mL} \\
<1,0 \mathrm{RU} / \mathrm{mL}\end{array}$ & $\begin{array}{l}<20 \mathrm{RU} / \mathrm{mL} \text { (negativ) } \\
\geq 20 \mathrm{RU} / \mathrm{mL} \text { (positiv) }\end{array}$ \\
\hline
\end{tabular}

sche Vaskulitis, Arzneimittelreaktion, Granulomatose mit Polyangiitis, urtikarielle Vaskulitis und andere viral bedingte Exantheme in Betracht.

Die Patientin wurde mit oralem Diphenhydramin, topischem Diphenhydramin, Triamcinolon 0,5\% Creme und Dexamethason 6 mg i.v. behandelt. Ihre häusliche Medikation (Metformin und Lisinopril) wurde während des Krankenhausaufenthalts unterbrochen, und wegen anhaltend erhöhter Blutzuckerwerte erfolgte eine Umstellung auf ein sliding-scale-Schema mit Korrekturinsulin. Lisinopril wurde aufgrund der Assoziation mit bullösem Pemphigoid ausgesetzt, obgleich die Patientin es seit Jahren eingenommen hatte ohne dass Probleme aufgetreten waren. Sie wurde unter oralem Dexamethason $6 \mathrm{mg}$ entlassen und schloss einen 10-tägigen Behandlungszyklus ab; zudem erhielt sie topisches Diphenhydramin und Triamcinolon. Wegen anhaltender Beschwerden im Zusammenhang mit dem Exanthem, die sich unter Steroideinnahme bessern, nach Beendigung der Steroidbehandlung jedoch wieder aufflammen, hat die Patientin seit ihrer Entlassung mehrere erneute Behandlungszyklen mit Steroiden durchlaufen. Darüber hinaus nahm Sie Nikotinamid ein, um ihr Exanthem zu lindern. Kurz nach ihrer Entlassung klangen die Dyspnoe sowie die Gliederschmerzen und das Fieber ab, und als einziges Symptom blieb das Exanthem weiter bestehen.

Die Patientin hat mehrere Steroid-Behandlungszyklen abgeschlossen, wobei weiterhin Exazerbationen des Exanthems auftraten. Ende November 2020 begann sie mit dem Ausschleichen 
der dritten Prednison-Behandlung, die sie seit ihrer Entlassung neben Doxycyclin erhalten hatte. Zum Zeitpunkt der Veröffentlichung wurden immunmodulierende Therapien in Erwägung gezogen, allerdings noch nicht eingeleitet.

Der Krankheitsverlauf seit der Erstvorstellung war wie folgt:

1. Erstvorstellung in der Klinik am 17.11.2020: Die Patientin erhielt Doxycyclin $100 \mathrm{mg}$ zweimal täglich und Nicotinamid 500 mg dreimal täglich und es wurde eine Prednison-Dosisreduktion eingeleitet. Wegen der COVID-19-Pandemie lehnte sie eine Behandlung mit Immunsuppressiva zunächst ab.

2. Telekonsultation am 9.12.2020: Entsprechend den Empfehlungen des Herstellers setzte die Patientin Doxycyclin und Nicotinamid ab.

3. Die Patientin wurde vom 11.12. bis zum 12.14.2020 wegen eines Schubs des bullösen Pemphigoids, der eine Hospitalisierung zwecks Schmerzkontrolle und Wundversorgung erforderte, stationär behandelt.

4. Praxisbesuch am 29.12.2020: Die Patientin hatte eine Verschlimmerung der schmerzhaften Blasenbildung im Gesicht, an den Armen, in der Leiste, an den Beinen und am Rücken festgestellt, die ihren Schlaf und ihre Arbeitsfähigkeit beeinträchtigten.

5. Die Patientin erhielt zwei Rituximab-Infusionen am 11.01.2021 und am 25.01.2021. Zur Behandlung des Pruritus nahm sie weiterhin Hydroxyzin 25-50 mg täglich zur Nacht ein.

6. Die Patientin stellte sich am 23.3. in der Hautklinik vor, und nach der zweiten Rituximab-Infusion geht es ihr sehr gut. Sie hat keine Blasen und die Predison-Dosis wurde auf 7,5 mg reduziert. Die Dosis wird weiter schrittweise verringert. In 4 Wochen wird die Patientin nachuntersucht.

Es wurde eine mündliche Einwilligung zur Verwendung der Bilder und des Fallberichts von der Patientin eingeholt. Eine schriftliche Einwilligungserklärung der Patientin wurde nicht eingeholt, da dieser Fallbericht keine identifizierbaren Daten enthält.

\section{Diskussion}

Dieser Beitrag enthält eine Übersicht über die aktuelle Literatur zu kutanen Manifestationen von SARS-CoV-2 und beschreibt den Fall einer Frau, die positiv auf COVID-19 getestet wurde, nachdem sie zwei Tage vor ihrer Diagnose ein anuläres, bullöses, juckendes Exanthem an ihren Extremitäten festgestellt hatte. Das klinische Bild ihres Exanthems war vereinbar mit einem bullösen Pemphigoid, einer Erkrankung, die bislang nicht mit COVID-19 in Verbindung gebracht wurde.

In einer kürzlich veröffentlichten Literaturübersicht berichteten Gisondi et al. über neuartige kutane Manifestationsformen von COVID-19 und beobachteten verschiedene dermatologische Erscheinungsbilder. Diese umfassten Exantheme (varizellenähnliches, papulovesikuläres und masernähnliches Exanthem), vaskuläre (chilblain-artige Läsionen, Purpura/Petechien und livedoide Läsionen) sowie urtikarielle und akropapulöse Eruptionen [2]. Unsere Patientin, die sich mit einem bullösen, juckenden Exanthem vorstellte, passt in keine der genannten Kategorien. Dies macht ihren Fall besonders ungewöhnlich, da bisher keine Daten über bullöse dermatologische Muster bei COVID-19-Patienten veröffentlicht wurden. Die am ehesten vergleichbare berichtete Erscheinungsform ist vielleicht die varizellenähnliche Manifestation, die kürzlich als mögliche frühe und recht spezifische Manifestation von COVID-19 festgestellt wurde [2].

Gemäß einer gängigen Auffassung über die kutane Beteiligung bei COVID-19 kommt es bei den betroffenen Patienten zu unterschiedlichen Formen von Vaskulitis, die sich unter anderem auf die dermoepidermale Junktionszone der Haut auswirkt. Becker untersuchte die Auswirkungen von COVID-19 auf das Kreislaufsystem und fand mehrere Fallberichte, in denen eine urtikarielle Vaskulitis beteiligt war [3]. In der histopathologischen Untersuchung von Biopsiematerial waren eine Dermatitis und Gefäßdegeneration der Basalschicht der Epidermis erkennbar. Daneben finden sich häufig eine Endotheliitis mit lymphozytärer Infiltration der dermalen Vesikel und Arteriolen sowie Mikrothrombosen der papillären Hautkapillaren [3].

Zudem liegen zahlreiche Fallstudien vor, in denen über dermatologische Erkrankungen im Zusammenhang mit COVID-19, die nicht unbedingt in die Kategorie Vaskulitis fallen, berichtet wurde. Aufgrund des schwierigen Zugangs zu dermatologischer Beratung während der Pandemie werden Hautmanifestationen der Krankheit möglicherweise unterschätzt, und es ist wichtig, sie weiter zu untersuchen.

Zwar sind COVID-19-bezogene dermatologische Erkrankungen in der Literatur relativ gut dokumentiert, doch wurde die Manifestation eines bullösen Pemphigoids wie bei unserer Patientin bisher nicht mit einer COVID-19-Infektion in Verbindung gebracht. Das bullöse Pemphigoid ist eine seltene Erkrankung, bei der es zu einer intraepidermalen Blasenbildung kommt und die durch Autoantikörper gegen Adhäsionsmoleküle in der Haut und den Schleimhäuten verursacht wird. Die Blasen bilden sich spontan und schmerzen, wenn sie platzen. Beim bullösen Pemphigoid handelt es sich um eine vergleichsweise gutartige, juckende Erkrankung, die mit prall gespannten, überwiegend auf der Beugeseite auftretenden Blasen einhergeht und deren Verlauf durch Exazerbationen und Remissionen gekennzeichnet ist [4]. Am häufigsten sind Patienten im Alter zwischen 40 und 60 Jahren von der Krankheit betroffen und Prodromalsymptome sind unter anderem Juckreiz, Dermatitis oder Urtikaria. Die Läsionen können lokalisiert oder diffus auf der gesamten Hautoberfläche auftreten, finden sich jedoch meist an den Beugeseiten der Arme und Beine sowie in den Achselhöhlen, Leisten und am Unterbauch [5]. Das Krankheitsbild unserer Patientin scheint zu diesem klinischen Bild zu passen, da es nach der initialen Behandlung mehrfach zu Exazerbationen kam und das Exanthem an Armen und Beinen, in der Leiste und am Rücken auftrat.

Bei einem Schub des bullösen Pemphigoids kommt es zu einer Bindung von Autoantikörpern an die Proteine von Hemidesmosomen, durch die ein inflammatorischer pathophysiologischer Prozess ausgelöst wird, der letztlich zu einer Spaltbildung in der dermoepidermalen Junktionszone und zur Blasenbildung führt [6]. Den Annahmen zufolge bilden sowohl infiltrierende Entzün-
198

Kompass Autoimmun 2021;3:195-200 DOI: $10.1159 / 000519460$ 
dungszellen als auch residente Hautzellen, darunter Keratinozyten, Fibroblasten und Endothelzellen, Zytokine und Chemokine, die am pathophysiologischen Prozess des bullösen Pemphigoids beteiligt sind [6]. Interessanterweise wird vermutet, dass bei vielen schwer an COVID-19 erkrankten Patienten eine der Hauptursachen für das Multiorganversagen mit einem Zytokinsturm zusammenhängt, bei dem übermäßige Mengen von Entzündungszellen Organschäden in Lunge und Leber verursachen. Nach einem in der Fachzeitschrift Mediators of Inflammation veröffentlichten Artikel gehören IL-1B, IL-17 und TNF- $\alpha$ zu den COVID-19-assoziierten Zytokinen, die auch bei Patienten mit bullösem Pemphigoid nachgewiesen wurden [6]. Diese Beobachtung könnte damit zusammenhängen, dass es bei Patienten mit kutanen Manifestationen der Krankheit zu einer bestimmten Form von Zytokin-Crossover kommt, und sie sollte näher untersucht werden, um ein tieferes Verständnis der Mechanismen im Zusammenhang mit der Entzündungsreaktion bei COVID-19 zu erhalten. Die Laboruntersuchungen bei unserer Patientin ergaben erhöhte Alanin-Aminotransferase (ALT)- und Aspartat-Aminotransferase (AST)-Werte, was auf eine Leberschädigung hinweisen könnte, sowie eine Erhöhung des C-reaktiven Proteins, das ein Marker für akute Entzündungsreaktionen ist. Die Interleukinspiegel wurden bei der Aufnahme nicht bestimmt, und da die Patientin während ihres gesamten Krankenhausaufenthalts fieberfrei war, gingen wir nicht davon aus, dass unsere Patientin eine durch einen Zytokinsturm bedingte systemische Entzündung hatte, wie sie bei manchen COVID-19-Patienten zu beobachten ist.

Es ist bekannt, dass die zugrunde liegende Ursache für das bullöse Pemphigoid Autoantikörper gegen Adhärenzproteine in der Haut sind; allerdings liegen nur wenige Studien vor, in denen die auslösenden Faktoren für die Bildung solcher Autoantikörper erörtert werden [7]. Nach Ansicht von Patel et al. ist die Ätiologie des bullösen Pemphigoids wahrscheinlich multifaktorieller Natur, wobei umweltbedingte Auslöser und die genetische Prädisposition eine Rolle spielen und die Komplementablagerung potenziell ebenfalls von Bedeutung ist, da in der Blasenflüssigkeit Komplement nachgewiesen wurde [7].

Es existieren Fallberichte über Patienten, die nach Impfungen und Virusinfektionen ein bullöses Pemphigoid entwickeln [8]. Einer verbreiteten Theorie zufolge führt die Einbringung eines Impfstoffs oder Virus zu einer zellulären Aktivierung von IL-17 und schließlich zur Freisetzung von proinflammatorischen Zytokinen und proteolytischen Enzymen, wodurch es zu einer Unterbrechung der Hemidesmosomen und Blasenbildung kommen kann [8].

Zwar wird in der aktuellen Literatur über eine starke Korrelation zwischen Virusinfektionen und der Entwicklung von BP berichtet, doch sollte bei den Überlegungen berücksichtigt werden, dass die meisten Patienten mit einem diagnostizierten Pemphigus-Exanthem eine genetische Prädisposition für die Entwicklung von Autoantikörpern gegen die Hautproteine BP180 und BP230 aufweisen, die für die Aufrechterhaltung der epidermalen Adhäsion von zentraler Bedeutung sind [7, 9]. Es ist wichtig daran zu den- ken, dass Menschen mit entsprechender genetischer Prädisposition bei Exposition gegenüber bestimmten Medikamenten, darunter DPV-Inhibitoren wie Vildagliptin und Linagliptin, bestimmte Diuretika wie Furosemid und Spironolacton sowie bestimmte Antipsychotika und Checkpoint-Inhibitoren, ein arzneimittelinduziertes BP entwickeln können [10]. Die zweite mit bullösem Pemphigoid assoziierte Wirkstoffklasse sind Angiotensin-Converting-Enzyme-Inhibitoren (ACE-Hemmer), insbesondere Losartan, Valsartan und Lisinopril [11]. Interessant ist, dass unsere Patientin Lisinopril eingenommen hatte. Eine PemphigusReaktion auf Lisinopril ist zwar selten, dennoch wurde das Medikament bei der Erstaufnahme der Patientin abgesetzt. Es ist unwahrscheinlich, dass ihre Symptome Folge der Medikamente waren, da die Symptome in der Regel nach Absetzen der Medikation abklingen, was hier nicht der Fall war. Dies ist auch deshalb weniger wahrscheinlich, weil die Patientin die Medikamente seit fast 20 Jahren ohne Probleme eingenommen hatte.

Nach Berichten aktueller Studien weisen fast alle Patienten, die einen Pemphigoid-Ausschlag entwickeln, eine erhöhte Frequenz des Allels DQB10301 auf, wobei einige Studien zeigen, dass die BP180-spezifischen Th1- und Th2-Zellen auf das Allel HLADQB10301 beschränkt sind [7, 9, 12-14]. Es sei darauf hingewiesen, dass bei unserer Patientin BP180- und BP230-Antikörper nachgewiesen wurden. Auch wenn bei unserer Patientin keine genetischen Untersuchungen vorgenommen wurden, ist es wichtig zu verstehen, dass ihr Genom zur Entstehung des Exanthems beigetragen haben könnte.

Ein weiterer wichtiger Gesichtspunkt bei der Ätiologie des BP ist die Bedeutung der Komplementablagerungen, die in der Blasenflüssigkeit nachgewiesen wurden, insbesondere bei unserer Patientin, bei der COVID-19 neu diagnostiziert wurde. Nach Angaben von Romeijn et al. sind in 83,1 \% der Hautbiopsien von Patienten mit diagnostiziertem bullösem Pemphigoid Komplementablagerungen in der Blasenflüssigkeit zu finden [15]. Dies gilt es zu bedenken, besonders in Anbetracht des Berichts von Java et al. über die Fähigkeit des Coronavirus, Komplementsignalwege zu aktivieren, und deren mögliche Beteiligung an der Schwere der Erkrankung [16]. Es wäre interessant, Genaueres über die Beteiligung der Komplementsignalwege bei BP und COVID-19 und über ihre gegenseitige Korrelation zu wissen.

Es sei darauf hingewiesen, dass es durchaus sein kann, dass bei unserer Patientin beide Erkrankungen gleichzeitig aufgetreten sind, ohne dass die eine der Auslöser für die andere war. Die Möglichkeit, dass die beiden Krankheitsprozesse bei dieser Patientin in keinem Zusammenhang zueinander standen, sollte in Betracht gezogen werden. Eine weitere Abklärung des ursächlichen $\mathrm{Zu}$ sammenhangs zwischen COVID-19 und BP ist erforderlich.

\section{Schlussfolgerung}

Die vorliegende Arbeit beschreibt eine ungewöhnliche dermatologische Manifestation von COVID-19 und stellt die aktuelle Literatur zur möglichen Pathophysiologie von BP bei COVID19-positiven Patienten vor. Das SARS-CoV-2-Virus gelangt über 
den ACE2-Rezeptor, der auf zahlreichen Zelltypen, darunter auch Hautzellen, zu finden ist, in die Wirtszelle. Das Eindringen des Virus in die Wirtszelle führt zu einer Komplementaktivierung, wodurch Entzündungszellen zu der Zelle rekrutiert werden und eine Reaktion ausgelöst wird [16]. Hinsichtlich einer Beteiligung an der Ätiologie des bullösen Pemphigoids gibt es Spekulationen, und dieser Fall liefert neue Hinweise auf COVID-19 als potenziellen Auslöser bei Patienten mit BP, die weiter untersucht werden sollten.

Diese Untersuchungen sind wichtig, damit Ärzte COVID-19 und die damit verbundenen dermatologischen Manifestationen besser verstehen. Die Pathogenese von COVID-19 und bullösem Pemphigoid ist komplex. Unter Umständen wird die Erforschung des Zusammenhangs zwischen den beiden, wie in diesem Fall, ein eingehenderes Verständnis der jeweiligen Krankheit und der Mechanismen, durch die sie entstehen und fortschreiten, ermöglichen.

\section{Patientenperspektive}

„Es war wirklich furchtbar und schwierig. Der Juckreiz und die Schmerzen waren schwer zu ertragen. Ich mache mir Sorgen, welche Folgen es hat, dass ich so jung an bullösem Pemphigoid erkrankt bin. Man sagt, es dauert Jahre, bis die Krankheit vollständig verschwunden ist, und normalerweise sind ältere Menschen davon betroffen. Es ist mir peinlich, wenn die Blasen auf meinen Armen sind und andere sie sehen können. Wenn COVID die Ursache ist, sollten die Leute wirklich den Rat befolgen und Menschenansammlungen meiden.

Wenn ich weniger als $30 \mathrm{mg}$ Prednison nehme, verschlimmern sich meine Symptome.

Es ist schwierig, nachts zu schlafen, weil die Blasen auf meinem Rücken so stark jucken und es schwer ist, darauf liegen. Die Krankenversicherung bezahlt nur eine kleine Menge an Cremes zur örtlichen Anwendung, und ich brauche so viel für den ganzen Körper, dass das keine echte Option ist. Manchmal helfen heiße Duschen gegen den Juckreiz, aber ich kann trotzdem nicht gut schlafen. Es ist einfach schwierig und ich hoffe, dass es besser wird."

\section{Erklärung zur Datenverfügbarkeit}

Der Fallbericht, die Metaanalyse und die wissenschaftlichen Daten, die zur Untermauerung der Ergebnisse dieser Studie herangezogen wurden, wurden in der vorliegenden Arbeit berücksichtigt. Die früheren Studien werden an den entsprechenden Stellen im Text als Referenzen zitiert [1-15].

\section{Genehmigung durch die Ethikkommission}

Diese Studie wurde vom Institutional Review Board (IRB) der Rocky Vista University genehmigt (RVU IRB Nr. 2020-0123 Exempt Category).

\section{Disclosure Statement}

Die Autoren erklären, dass keine Interessenkonflikte bestehen.

\section{Danksagung}

Die Autoren danken Kelsey Sherman, Bradley Brown, Jonathan Dill, Kimberly Insel, Trevor Harrington, Tom Califf und Zoey Thompson für die Versorgung ihrer Patientin und für die informativen Diskussionen, die zur Erstellung dieses Manuskripts beigetragen haben. Die Bilder wurden von Tom Califf und Angela Delano bereitgestellt.

\section{Lizenzangabe}

Olson N, Eckhardt D, Delano A: New-Onset Bullous Pemphigoid in a COVID-19 Patient. Case Rep Dermatol Med. 2021;2021:5575111 (DOI 10.1155/2021/5575111). ${ }^{\odot} 2020$ The Author(s). (Übersetzung), lizensiert unter CC BY 4.0 (https://creativecommons.org/licenses/by/4.0/deed.de).

\section{Literatur}

Die Literatur ist unter www.karger.com/doi/10.1159/000519460. 